\title{
A Test-Bed for Emergency Management Simulations
}

\author{
A case-study including Live Call Data in New Zealand
}

\author{
Anu Vaidyanathan \\ Department of Computer Science and Engineering \\ Indian Institute of Technology Ropar \\ Nangal Road, Rupnagar India. \\ anu@iitrpr.ac.in
}

\begin{abstract}
We present a test-bed for Emergency Management Simulations by contrasting two prototypes we have built, CAVIAR and Reverse 111. We outline the desirable design principles that guide our choices for simulating emergencies and implement these ideas in a modular system, which utilizes proactive crowd-sourcing to enable emergency response centers to contact civilians co-located with an emergency, to provide more information about the events. This aspect of proactive crowd-sourcing enables Emergency response centers to take into account that an emergency situation's inherent nature is dynamic and that initial assumptions while deploying resources to the emergency may not hold, as the emergency unfolds. A number of independent entities, governmental and non-governmental are known to interact while mitigating emergencies. Our test-bed utilizes a number of agents to simulate various resource sharing policies amongst different administrative domains and non-profit civilian organizations that might pool their resources at the time of an emergency. A common problem amongst first responders is the lack of interoperability amongst their devices. In our test-bed, we integrate live caller data obtained from traces generated by Telecom New Zealand, which tracks cell-phone users and their voice and data calls across the network, to identify co-located crowds. The test-bed has five important components including means to select and simulate Events, Resources and Crowds and additionally provide a visual interface as part of a massive online multi-player game to simulate Emergencies in any part of the world. We also present our initial evaluation of some resource sharing policies in our intelligent agents, which are part of our test-bed.
\end{abstract}

Keywords-test-bed, Emergency Management, Live Call Records, PCMD, Proactive Crowd-Sourcing, Agents

\section{INTRODUCTION}

Simulations in the field of Emergency Management, which adapt an end-to-end approach $[40,41]$ are important for a number of end uses including training personnel, understanding how administrative boundaries affect resource sharing and analyzing how to optimize resource-allocation and response time. Improving the efficiency of first responders is of greatest importance to response teams. Evaluations of operational alternatives are also of interest to these teams and can be easily programmed into a simulation. Normally, in the case of an Emergency or Disaster, several disparate teams work in unison to mitigate the situation at hand. This diversity in administrative units demands a dynamic system that is able to represent various entities and the resources they are able to make available to the situation. Evaluating network connectivity during an Emergency helps estimate available modes of communication with those affected and between groups of first responders. In our work, we introduce a layer to indicate how the infrastructure of Telecom New Zealand, specifically their cell-phone towers are laid out and how they handle calls during periods of high call traffic. We envision this to assist us in decision making in the case of an Emergency, during which time the call-traffic patterns are also high in volume. Dynamic Data Driven Application Systems (DDDAS) have been discussed to have the end goal of processing real-time experimental data to use as an input parameter for software simulations [1]. Since experimental data often requires simulations to make drastic changes [2], the end-goal for a good simulator has always been flexible design. Complex Adaptive Systems (CAS) and Agent Based Models (ABM) have also been proposed for modelling Emergency Operations Centers [3]. GEMASim models EOC operations by abstracting entities such as the SOC Chief, the Public Affairs Officer, the Operations Chief etc. into their own individual agents, to represent different functionality. A limitation of adhering too closely to prescribed service hierarchies is the lack of inclusion when it comes to local civilian groups. Integrated Gaming and Simulation for Incident Management has been proposed [4] wherein different modules exist for Plume Simulation, Crowd Simulation, Traffic Simulation (of social behaviours and vehicular traffic), health-care simulation, transportation simulation and an integrated gaming module for evaluating incident management. This does not introduce any network backbone or evaluate inter-operability of devices handled by first responders, each often working in its own frequency band [6].

\section{A. Related Work}

The Pacific Rim Visualization and Analytics Center coordinated at the University of Washington, known as the 2 PARVAC to other regional centres, has been working on developing improved shared artefacts for communicating during community-wide first response activities [8]. The use 
of sensors for Emergency Medical care is probably the most wide-spread. Bluetooth compatible devices have been studied in great detail to assimilate data and information and interface to a central server by means of a cellular phone $[9,10,11]$. Sensors are also used in the case of emergency evacuations [12] from within buildings. Emergency response simulation using sensors have been performed [13] in order to co-ordinate and control the dispatch and use of resources required. Specialized computation of emergency exit routes in case of toxic gas attack [14] have been discussed where models are used to compute the dispersion of the gases. Here the sensors are those used by the live weather web service which is able to predict and supply weather patterns for any zip code provided. The use of computers to control and co-ordinate emergency response have evolved from using static computer $[15,28]$ to primarily using mobile sensors $[16,17,18,21,27]$. The use of cellular phones [ 23,24$]$ has been mentioned in a few pieces of work however, there is no integrated solution for end-to-end disaster response or emergency management. The development of serious games for a variety of purposes extends across utility including role-playing in the case of Emergencies [32,37]. Several requirements exist for such games including a valid environment in which to train, practice and evaluate co-ordination amongst distributed entities, amongst various administrative domains. In general, these games can be easily extended to being simulations [ 40 , 41] when played in single-player mode and therefore extend in functionality to studying performance of various approaches to live emergency response. The background of this work lies in a body of work that looked at early warning systems [38] which relied heavily on a prediction-based model to issue warnings to affected areas. In an Emergency System with online simulation models [36] that take input from sensors that measure environmental variables can be used by emergency services. [36] proposes an approach to establish information exchange and communication among many subsystems using a High Level Architecture. This work does not utilize live call information from telephony service providers as a layer in its visualization and is constrained in its ability to accept userinput to the system to simulate how first responders behave at the time of an emergency. User-Centered Design [35] is proposed in an All Hazard Exercise Development and Administration tool for emergency management agencies, once again to incorporate training elements in the simulation. The utility of knowledge sharing has been propounded in many works $[33,42,43,34,44,45]$ The sensors proposed in these cases are very resource constrained in terms of hardware and not connected by any service-provider's network. In the case of response rather than warning, the work in the realm of simulations or games is rather sparse. Previous work on wireless infrastructures designed to address emergency scenarios while allowing intercommunication between teams in a scenario with existing network infrastructures and the pervasive grid network [39] do not propose citizen participation in the process of mitigation. Furthermore, the use of cell-phone data for emergency response is proposed in this work for the first time. Contacting callers on the ground by using the concept of proactive crowd sourcing is also proposed in order to assimilate accurate, real-time information about how the situation on the ground is changing, as an emergency evolves. By enabling modular design for rapid feedback from co-located callers, emergency response centers can better decide how to deploy resources to the emergency.

\section{DESIGN PRINCIPLES FOR A TEST-BED FOR EMERGENCY MANAGEMENT}

In this section, we discuss some of the desirable characteristics in a simulation environment, specifically for Emergency Management and Response, which have affected our design principles.

A. Evolution of an end-to-end design: The continuum of emergencies starts with the occurrence of the emergency or early warning systems (depending on predictors being set up), the assimilation of information and data pertaining to various aspects of the emergency, the prevention of the emergency if possible and responding to the emergency. Depending on the nature of the emergency, a variety of tools and techniques might render themselves relevant. The emergencies themselves could belong to a wide category including natural hazards, civil emergencies, medical emergencies, epidemics and large-scale terrorist attacks. The parts of the continuum in which the sensors are used are also varied. For example, certain sensors are used to detect the occurrence of an emergency. Other sensors might be used to assimilate data and information about the emergency, requiring them to have a higher hardware specification, when compared to simple sensors. Sensors can also be used to simulate emergencies, issue early warnings to people affected by the emergency and mitigate emergencies. Fig.1shows the continuum of an emergency situation.

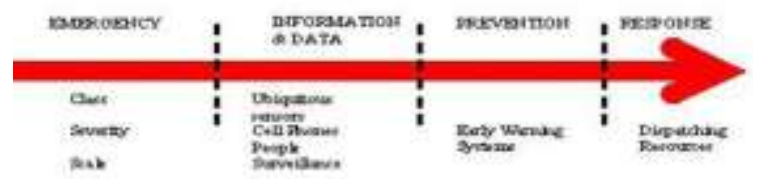

FIGURE 1. Emergency Continuum. Different phases exist within emergency management including occurrence, surveillance, prevention and response.

\section{B. Ability to incorporate a number of governmental and} non-governmental entities and policies: Some observations on recent disaster statistics including the Leh Flash Flodds, the Christchurch earthquake [] and an older example in Hurricane Katrina [] indicate that governmental response agencies co-ordinate response activities with civilian groups to mitigate the situation. Therefore, it is important for any simulation tool to be able to incorporate both governmental and non- 
governmental entities and policies in its framework. Related work [HLA Based] proposes methods to simulate tasks within an EOC while adhering to IEEE standards on distributed simulation systems. Our work keeps with this theme of separating the run-time architecture into Software Simulations, Live components and data-viewers while extending the architecture to be able to make decisions based on interactions with non-governmental agencies and common citizens via proactive crowdsourcing.

C. Efficient, inter-operable communication models: One of the earliest limitations identified with first-responder communications was that they were largely outdated and exposed a very big weakness in operational efficiency of these teams, especially in the case of large-scale disasters. In the US alone, there are approximately 50,000 local first responder agencies, a large number of which struggle with establishing communication channels with each other. Project 25 was one of the earlier efforts that tried to integrate groups working in the domain of Public Safety to identify issues with their devices and channels to contact each other and co-ordinate response activities. With consistent effort, P25 has evolved to assessing the availability of reliable wireless communication for these responders and introduced a variety of standards including Land Mobile Radio (LMR), services for local, state and federal public safety agencies [7]. A number of commercial service providers have also stepped in to take the lead in addressing this problem including Cyren Call, the First Response Coalition and Frontline Wireless. A good simulation test-bed must take into account varying connectivity levels in order to realistically model coordination between distributed teams.

D. Ability to change response parameters with live feedback: The inherent nature of an Emergency is dynamism and any good simulation test-bed, whether it is used for training personnel or evaluating response mechanisms, should take into account the fact that assumptions about the parameters change, with time. For example, a resource might be trying to traverse a path which crosses a bridge and if that bridge falls down during the emergency then that path is no longer valid, when simulating the approach of the resource dispatched for that emergency.

\section{SimUlATORS FOR EMERGENCY MANAGEMENT}

Our initial approach to simulating Emergency Response was captured in a somewhat static framework to demonstrate user-mobility at the time of an Emergency, in a prototype CAVIAR. This design was very limited for a variety of reasons, including the infeasibility of adding new information in a layered fashion, defining boundaries of various administrative domains and encoding the notion of time in a more intuitive manner. In order to understand user-mobility and plot where the cell-phone tower resources were located, we initially used OpenSceneGraph along with static terrain maps of the South Island. The system to map user-mobility in our initial simple visualization of call loads and user movement is shown in Fig. 2.

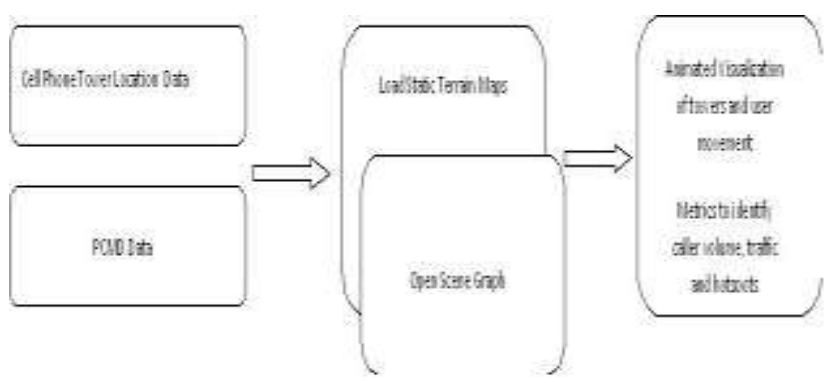

FIGURE 2. Static Visualization - CAVIAR. Our Initial Prototype included a lot of static components such as terrain maps, only two sources of data (tower location and PCMD) and produced animated visualizations of user-mobility and tower-load.

We evolved our initial design with CAVIAR to using a modular approach in building a simulation test-bed where all the characteristics listed in Section II are taken into consideration, including:

Event Simulators for simulating the occurrence of geographically distributed disasters.

- Resource Simulators which place various resources such as fire-trucks, ambulances, etc. at their appropriate locations.

- $\quad$ Crowd Simulators which position callers on a cell-phone network at their location during the occurrence of the emergency.

- Incorporation of agents for decision making. and

A run-time framework that is dynamic in accepting live caller feed-back on the changing nature of the emergency.

These elements are constituted in our prototype called Reverse 111 which is a part of a larger test-bed for Emergency Management Simulations. The overview of the test-bed is shown in Fig. 3. Reverse 111 has custom menus to simulate emergencies in any part of the globe wherein the NASA World Wind virtual globe is an integral part of our system. The NASA World Wind virtual globe is part of an opensource initiative where USGS satellite imagery is overlaid with several maps from ariel photography and other publicly available GIS data on 3D models of the earth and other planets. Several add-ons are available as part of this software including point layers, trial layers, line and polygon features, model features, place names, image layers and scripts. 


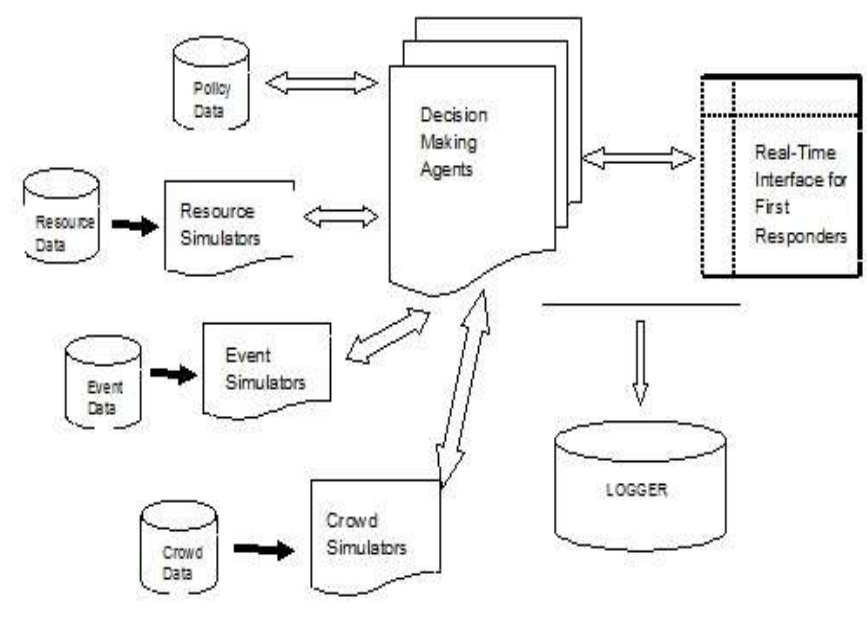

FIGURE 3. Emergency Response Test-Bed This figure shows the modules in our test-bed with emphasis on incorporating various sources of data and information.

In our architecture, we have special configuration files to simulate various scenarios within the emergency management framework. All emergencies are not created equal and for example, an earthquake will lead to a clustering of emergency events along a fault line, vs. a bio-weapon attack will have a different distribution of events as compared to a tsunami, where the events will be clustered along the shore. Additionally, we are able to dynamically configure invalid paths such as resources being unable to traverse over water bodies or gorges or roads that are blocked as a consequence of the event. Since these configurations are dynamic we enable constant feedback (for example from mobile phone users that are being proactively crowd-sourced for information) to modify the parameters within which our resource scheduling and planning has to work.

\section{A. Event Selection}

In our initial prototype with CAVIAR, event selection was performed by polling the busiest traffic days, as observed by the telephony service providers, Telecom New Zealand, one of the largest in the country. By characterizing call traffic and user-movement over the course of a special-event day, we successfully compared how call traffic differs between a normal day in the week versus a day on which an All-Blacks Rugby game was played in Christchurch. The data-sets used for the examples shown in Table1. The data was collected in hour-long blocks for our visualization.

TABLE 7-1. Data-Sets used to show call-traffic. we compare traffic over 24 hours, on two days, a normal day and the day and All-Blacks Rugby game is played in Christchurch.

\begin{tabular}{|c|c|c|c|}
\hline Trace & Date & Event & $\begin{array}{c}\text { Sample } \\
\text { Size }\end{array}$ \\
\hline $\begin{array}{c}\text { One day dataset } \\
\text { (Christchurch) }\end{array}$ & $\begin{array}{c}\text { 22nd } \\
\text { January } \\
2008\end{array}$ & $\begin{array}{c}\text { Norma } \\
\text { 1 Day }\end{array}$ & 1272184 \\
\hline
\end{tabular}

\begin{tabular}{|l|c|c|c|}
\hline $\begin{array}{l}\text { One day dataset } \\
\text { (Christchurch) }\end{array}$ & $\begin{array}{c}21 \text { st } \\
\text { June 2008 }\end{array}$ & $\begin{array}{l}\text { All } \\
\text { Blacks } \\
\text { Rugby } \\
\text { Game }\end{array}$ & 1667914 \\
\hline
\end{tabular}

Fig. 4 shows the call traffic within Christchurch, from the first data-set shown in Table 1. The data is broken up into hour-long samples, for one whole day, January 22nd, 2008. This is a normal weekday in the city with no special event occurring anywhere to create anomalous traffic patterns. Each tile represents an hour, starting at $8 \mathrm{am}$ with 12 tiles being displayed for 12 hours of the day from $8 \mathrm{am}$ to $7 \mathrm{pm}$. The visualization is straightforward in that the density of calls is depicted by the size and the brightness of the circle depicting that statistic, which is placed over that particular cell-tower, in the city.

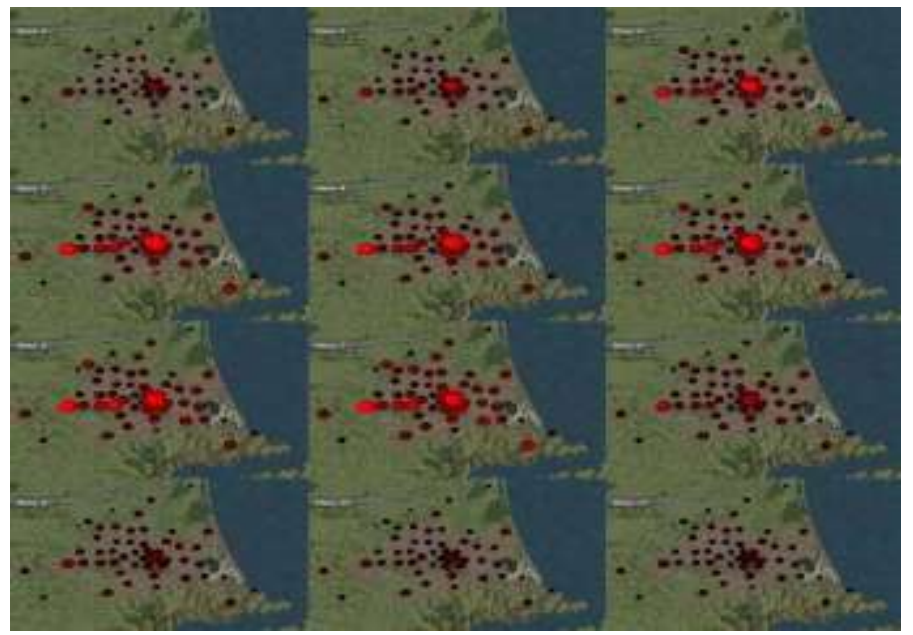

FIGURE 4. Normal Day Call Traffic. Call traffic is depicted within Christchurch on January $22^{\text {nd }}, 2008$, a normal day, over 12 hours.

As can be seen from Fig. 4, at 8am, the city wakes up and from $9 \mathrm{am}-5 \mathrm{pm}$, the city is most active, which pertains to the work-hours of people residing in the city. Also seen is the concentration of calls in the cell towers closers to the city centre, which is starting to brighten (depicting greater number of calls) at around 9am, which is the second tile in the topmost-row of tiles in Fig. 4. At around lunch hour $12 \mathrm{pm}-$ $1 \mathrm{pm}$, we see that the traffic starts to move to locations outside the city, possibly commuting to get lunch at spots outside the city-centre. This is depicted in the third tile in the second row. After about $5 \mathrm{pm} 5: 30 \mathrm{pm}$ the calls drop off as night-time approaches. 


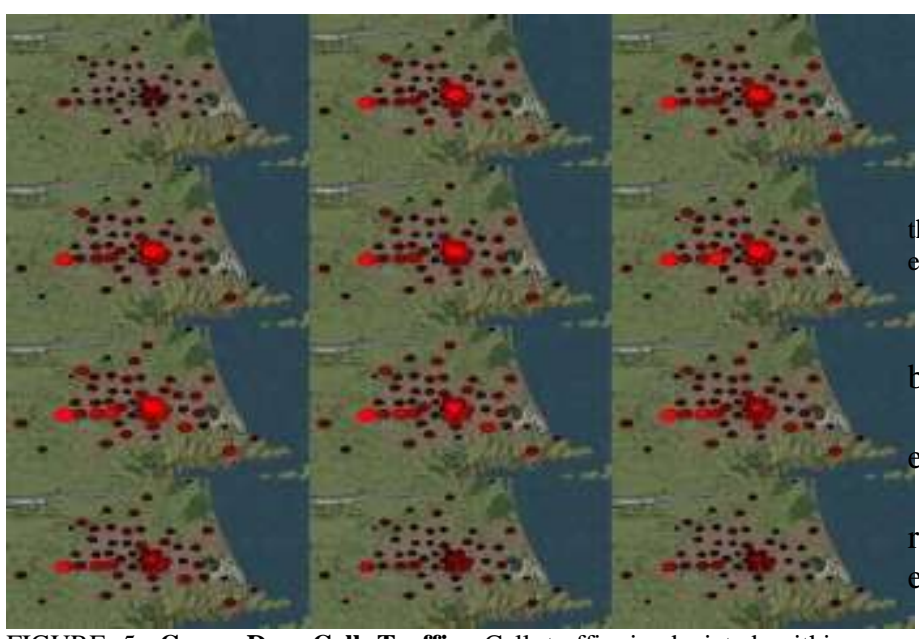

FIGURE 5. Game Day Call Traffic. Call traffic is depicted within Christchurch on June $21^{\text {st }}, 2008$, when an All-Blacks Rugby game was played, over 12 hours.

Fig. 5 shows the data collected for calls made on June 21st, 2008, corresponding to the date of an All Blacks game vs. England. The difference in call traffic here depicts how the city behaves during a holiday or during a special occasion. At 9am, the city is awake but not buzzing, as on a regular weekday, which was January 22nd, 2008 (a Tuesday). Furthermore, three is no migration of call traffic because most users have decided where they are going to be, at the time of the game, so the intensity stays static between tiles 3-8, depicting the hours of $10 \mathrm{am}-3 \mathrm{pm}$. At the time of the game being played, 7:35pm was the telecast time, depicted in Fig. 5, in tile 1 in row 1 and for a few hours preceding that, depicted in Fig. 7-7, tiles 1-3 in row 4 (showing $5 \mathrm{pm}-7 \mathrm{pm}$ ), the call traffic dies out as people are more focused on the game. This, in contrast to the same tiles 1-3 in row 4 (showing $5 \mathrm{pm}-7 \mathrm{pm}$ ) in Fig. 4, is much less call traffic. In Fig. 5, between the hours of $5 \mathrm{pm}-7 \mathrm{pm}$ on a weekday, the city was still pretty awake and call traffic depicted that. we have co-related this observation to other AllBlacks games played in Wellington and Auckland but do not include these for length restrictions.

\section{B. Event Simulators}

In Reverse 111, events refer to emergencies that are occurring in an area, that are represented in our system by means of icons appearing at certain latitude, longitude combinations over a geographic area. The icons chosen for these represent the resource requirements for these events. There are four different types of resources an event could need, and this is indicated by different colors. Figure 6 shows the iconic depiction of an event in our system. Examples of these include fire-trucks, medical ambulances, police-vehicles or military support. In Reverse 111, an event does not have fixed resource requirements.

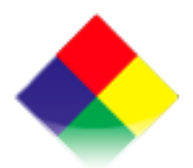

FIGURE 6. Event Depiction. We show the iconic depiction of an event in his figure wherein each color stands for a different type of resource that an mergency event might require.

The attributes associated with each event are outlined below:

Event Location refers to the actual physical location of the event;

Event Static Resource Requirements describes the static esource requirements that are assigned upon the creation of very event;

Event Dynamic Resource Requirements describe the changing nature of an event with the progress of time. While I borrow from the RimSim Response depiction, I extend an event's resource requirements to be more dynamic. In the original RimSim Response, an event is static in its resource requirements and this is not a realistic depiction of any emergency, where the needs are constantly changing over time. Additionally, the objective of Reverse 111 is not training (as was the case with RimSim Response), rather it is to make a case for obtaining better information from citizens or cellphone users that are co-located with an emergency. Therefore, our system needs to model an emergency event more realistically in order to support end-to-end evaluation;

Event Changing Access Parameters describes how the areas surrounding an event might change over time, given that our initial assumptions about an emergency may not be valid as time goes on.

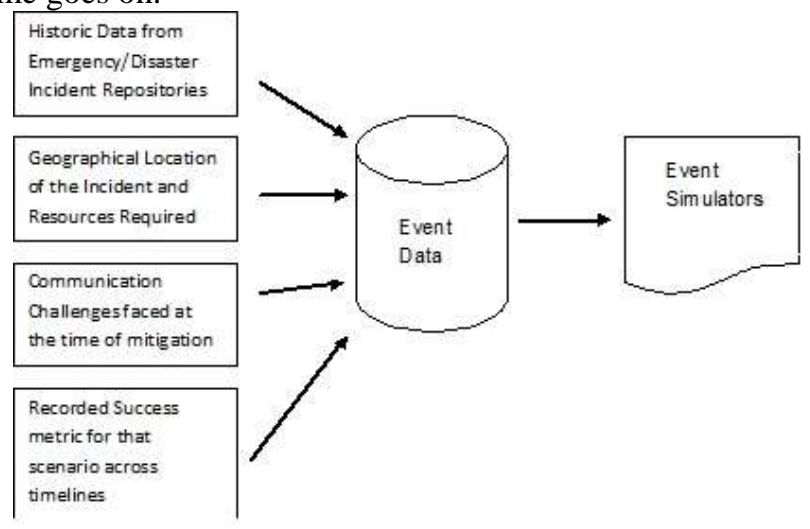

FIGURE 7. Event Simulators. Several data-sources are used as inputs to the Event Simulators including historic data from first responder repositories, in our test-bed.

Events occur at pre-determined locations or according to various pre-simulated scenarios. Fig. 8 shows the occurrence of three different emergencies within Seattle, including an earthquake, a bio-weapon attack and a tsunami, which have 
different distributions and groupings of events along the geographic spread of the region.

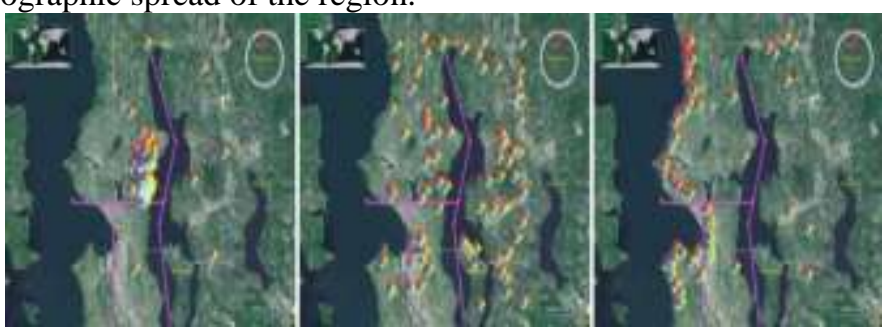

FIGURE 8. Different Event Distributions. Three different types of events including a bio-weapon attack, an earthquake and a tsunami show different occurrence and grouping of incidents in the greater Seattle area.

\section{Resource Simulators}

CAVIAR had no resource simulators. In Reverse 111, Resources refer to the resources that are applied in order to ameliorate the emergencies. Fig. 9 shows the types of resource used in Reverse 111. Examples include various state services and every resource has a number of attributes detailed below:

Resource Location refers to the geographical location of a resource. For example, a fire-truck might be at a fire-station when the emergency event occurs or might be returning to its home-base and therefore at a different location at the absolute time-stamp when its required.

Resource State indicates whether the resource is stationary or is in motion towards the event of interest.

Resource Owner refers to which administrative domain owns the resource. These could include state bodies such as the fire-department, police, military and can also be extended to include commercial emergency responders, which motivates our lottery-scheduler to evaluate a heterogeneity in such resource ownership.

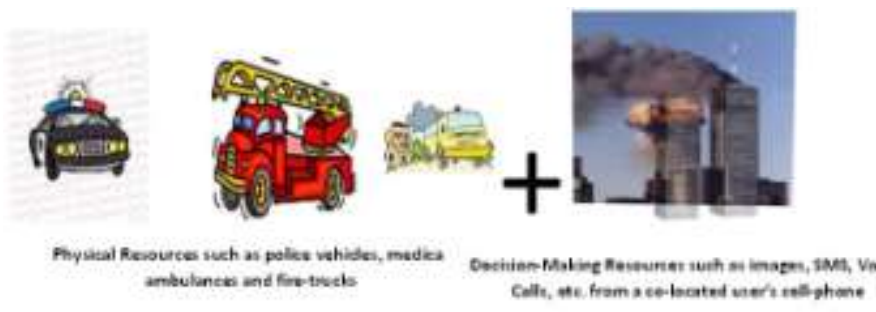

FIGURE 9. Resource Depiction. Physical resources are static and decision-making resources are more dynamic in our system.

Resource Type refers to whether the resource is a static resource (such as the ones described before) or a more dynamic resource, such as a user providing images of the façade of the building that has fallen down. The latter is more information than an actual physical resource and can be used for decision making. This is separate from the physical resources but an integral component of Reverse 111 .

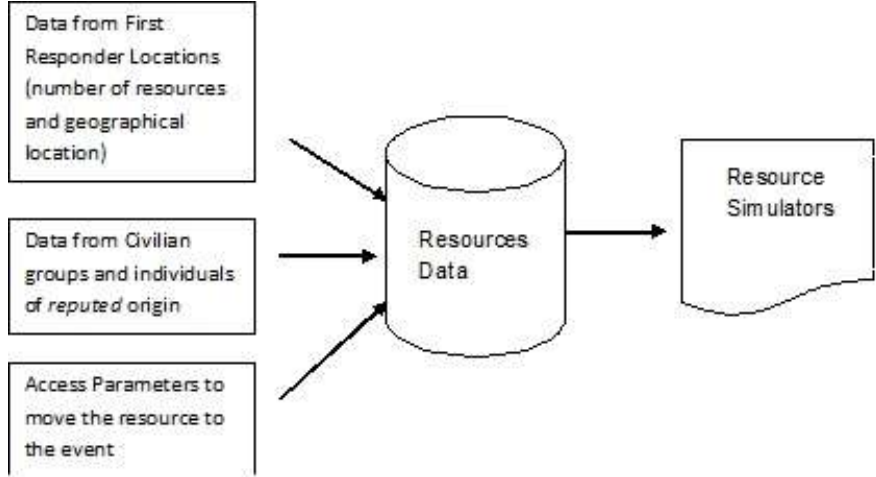

FIGURE 10. Resource Simulators. Physical resources are static and decision-making resources are more dynamic in our system.

\section{Crowd Selection}

Utilizing our first attempt at the design, we come up with a simple visualization of the cell phone towers (shown in Fig. 11 tower-statistics (shown in Fig. 12) and user-mobility (shown in Fig. 13) within CAVIAR.

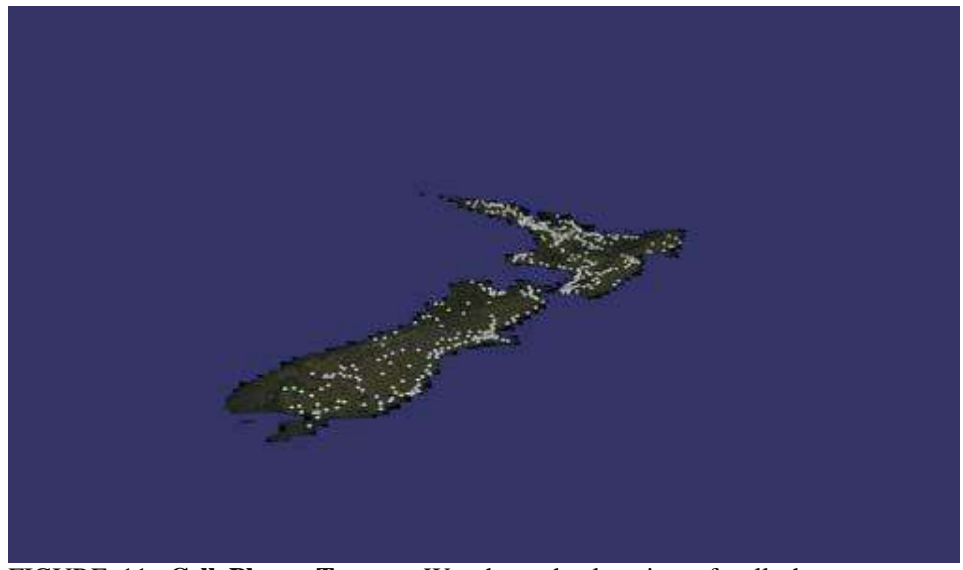

FIGURE 11. Cell Phone Towers. We show the location of cell-phone towers in this figure, from our simple visualization tool, CAVIAR.

Cell phone tower locations are contained in their own data set outside of PCMD. We are provided with unique identifiers for each of the cell phone towers and a (latitude, longitude) for each tower. We convert these into (Easting, Northing) for each tower. Easting and Northing are terms to describe what are essentially geographic Cartesian pairs for any point. Taking a base map of New Zealand, we plot these points on that map in order to depict the location of the cell-phone towers. It is easily observed that the North Island has a lot more towers than the South Island, owing to a larger subscriber base. Added to this, the grouping of towers is also indicative of the underlying terrain, for example, in the South Island, there are not too many towers in the regions housing the Southern Alps. This is not obvious from this visualization, however as there are no terrain depictions of the region. 


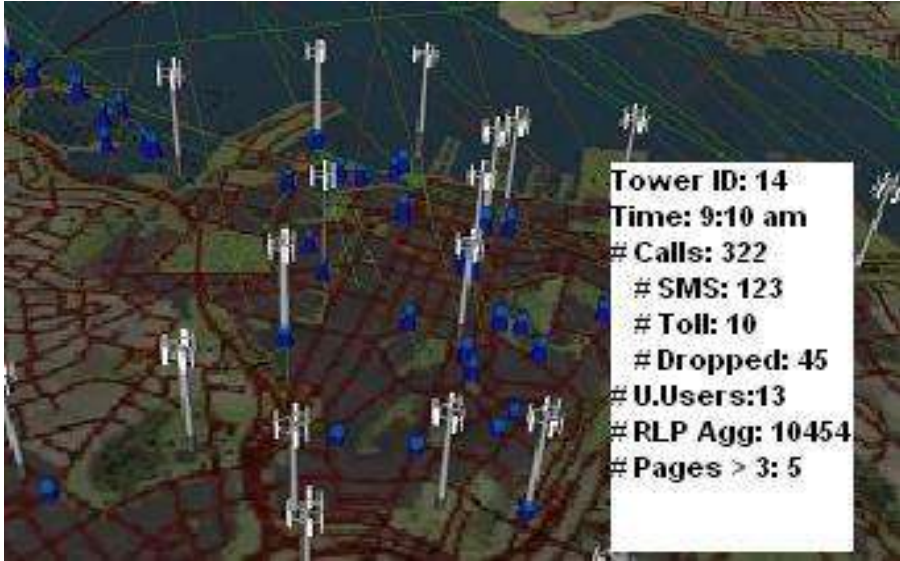

FIGURE 12. Tower Statistics. Cell Phone towers are the white structures, each of the users around the tower being depicted in blue. On rolling over a tower, simple statistics about the tower such as the Tower ID, the Time at which the query is being processed and other data related to the tower is displayed.

In CAVIAR, we depict phone subscribers with blue icons and enable basic zoom and roll-over functions and Fig. 12 shows the zoomed-in view where the user has rolled over a tower and the basic tower statistics are displayed to the user. In our particular example, the statistics we show the user (in this case, an emergency responder or a network capacity planner) such variables as the Unique ID of the tower, the time-stamp which the data-shown pertains to, the number of calls made, the different kinds of calls made (SMS, Toll calls, dropped calls), the number of unique users whose calls originated on the tower, the number of paging requests seen (which could provide insight into security violations on a tower) etc. The proximty of the subscirber to the tower is calculated using the call duration and the call start time and the ending cell tower. This is a static snapshot at 9:10am and does not take into account how the user is moving. Further, the orientation of the user is approximated based on movement and if the user is static, we can only estimate the distance from the center of the cell-tower.

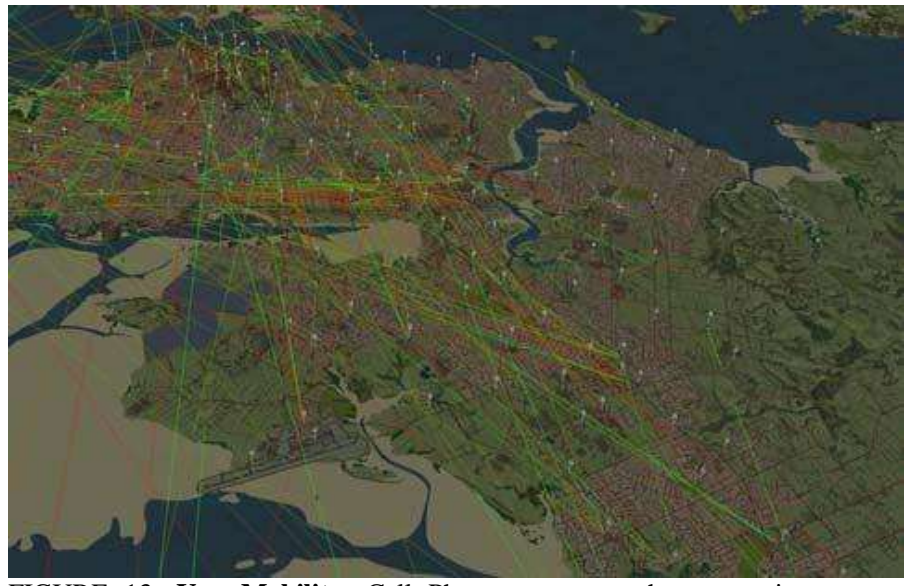

FIGURE 13. User-Mobility. Cell Phone users are shown moving at different rates (green being slowest, red being fastest and yellow and orange representing the range in between). This visualization is not effective in encoding time.
Fig. 13 shows user-mobility between cell-phone towers. In this example, we have filtered out the users that are moving between more than 2 cell-towers in the course of their conversation. By further filtering the data to only include a few minutes worth of calls, we are able to depict the users moving, along a certain trajectory (shown by green, orange and yellow lines) on the map. A user's velocity is calculated based on the starting and ending tower for a particular call, the length of the call and the location of the starting and ending towers. We depict the shortest path that a user can follow, during the course of the call. This is for purposes of visualization only as the user might well take a circuitous route. Added to this, our depiction of the user's movement does not address some of the infeasible paths such as traversing what are obviously water-bodies (shown in the top left-hand corner where we see users freely zooming over Lake Taupo while placing a phone call). For the initial studies on user-mobility, this depiction is sufficient for estimating call traffic and call volume as we will observe in coming examples.

\section{E. Crowd Simulators}

In Reverse 111, crowds are simulated by extending the crowd selection in CAVIAR to further incorporate the fourth design principle of changing response parameters based on live feedback. Crowds are introduced as a layer in Reverse 111 wherein the system is able to co-locate these crowds with the emergency event being simulated and poll them for more information with respect to the emergency. Proactive Crowd Sourcing involves the following steps:

1) Identifying co-located callers that are closest to an emergency event. In our system callers are identified by the cell tower from which their call originates at a time closest to the occurrence of an emergency. A group of callers located within a radius of $20-30 \mathrm{~km}$ around the emergency area are similarly identified with the intention of using information that they may provide to decide on how best to mitigate the emergency.

2) Calling co-located callers where the Emergency Response Center contacts the crowd identified in Step 1 and polls them for information (potentially voice confirmation of the emergency, an SMS on how things are unfolding, a picture to show the changing nature of access paths to get resources to an emergency etc.).

3) Assimilating relevant information in various media types and processing the same for more accurate response to the situation at hand.

Reverse 111 further has the ability to avoid invalid paths assuming that an access bridge has fallen down during the time the response center springs into action for both resources and the crowd, unlike our initial work with CAVIAR where the shortest path was used to calculate how users move. 


\section{A FLEXIBLE TEST BED FOR EMERGENCY RESPONSE SIMULATION}

Within our test-bed for emergency response simulation, we have placed emphasis on adhering to the four design principles outlined in Section II. This prototype test-bed has been developed as a massive online multi-player game, in order to involve more than one player (or response policy) when simulating emergencies. In this section, we describe our modular implementation wherein:

1) Evolution of end-to-end design is achived by evolving the toold from a static framework within CAVIAR to a more dynamic framework within Reverse 111.

2) Ability to incorporate a number of governmental and non-governmental entities and policies is achieved by first demarcating the region in which the simulations are performed into various administrative boundaries (such as towns or muncipalities, in our case into the cities of Christchurch, Rangiora, Oxford and Ashburton in one example on the South Island). This is followed by drawing fences to ensure that resource-sharing across these administrative domains is governed by local-policies. The third step is to introduce a number of agents for resource scheduling including a greedy agent [], a lottery agent [] and a round-robin agent [] to simulate the interaction between governmental and nongovernmental entities that might be pooling their resources and information to mitigate the emergency.

3) Efficient, inter-operable communication models are simulated by introducing cell-phone callers from Telecom New Zealand as an extra layer in the system. Since these work on the same frequency and provide broadband facilities where needed, we rely on a mode of communication that can be transparent across response teams. Within callers, there are high-priority callers (such as response teams) and low-priority callers (such as citizens trying to contact each other) and this stratification helps achieve channeling the important communication to the respective parties during emergencies.

4) Ability to change response parameters with live feedback by introducing proactive crowd-sourcing where we identify callers co-located with the emergency and the simulator introduces delays to simulate the Emergency Response Center contacting the co-located caller and receiving information from them to assess the best response mechanism.

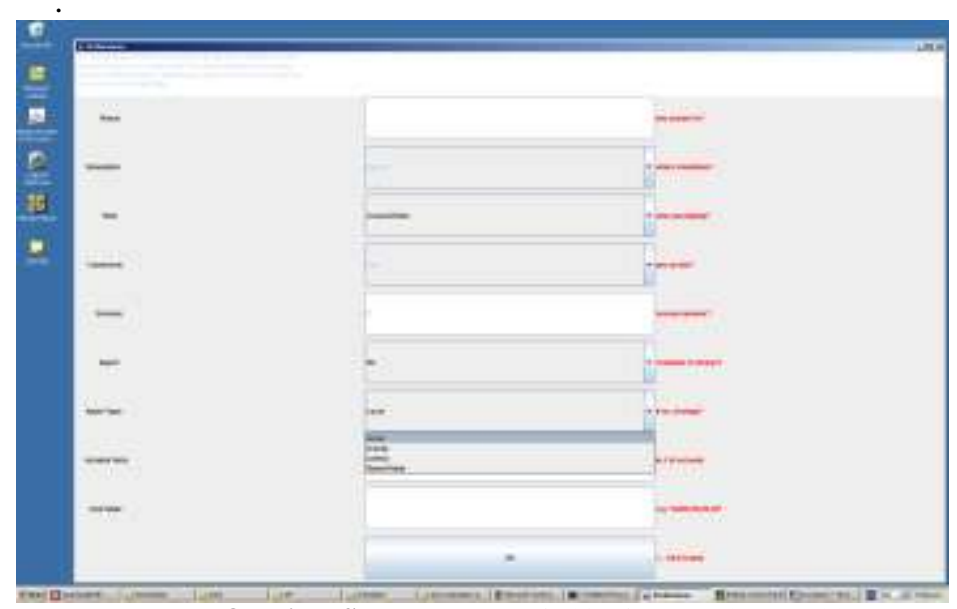

FIGURE 14. Opening Screen. Users of this framework are able to configure a lot of system parameters on the fly including roles assumed, playing with other online players, recording the history of their session, playing as human players or invoking scheduling agents that evaluate the effectiveness of various scheduling ideas, controlling the incident rate, etc.

Fig. 14 displays the opening screen of our prototype testbed showing the parameters that can be dynamically configured in the test-bed starting with the region which the user wants to simulate Emergency Management in, which could include any geographic region in the world. For purposes of example (where emergency situations can assume two different scales, we limit our discussions to New Zealand and the city of Seattle in the state of Washington, in the United States). The prototype further allows user to run the simulation as an oracle player who controls all administrative domains (which is useful for baseline comparisons) or as a particular administrative domain (such as the mayor of Christchurch), in order to understand which policies work best, given their resource constraints. There is a further provision to allow multiple-players connected online to play simultaneously, and is a powerful instrument for training first responders. The user's actions and findings can be recorded on a per-session basis, creating a powerful tool for analyzing historical results and comparing these with moving the parameters of the simulation around. Different types of resource-schedulers (or agents) can be evaluated in this tool and in case the user does not want any agent, wants to schedule resources themselves then they can play as themselves, human users. Users are also able to choose the rate of occurrence of emergencies (once more, the emergencies will have a notion of scale associated with them and an emergency in India affects a lot more people and occurs quicker and more frequently if wide-spread, as opposed to an emergency in New Zealand) to keep our results realistic, when evaluating various approaches and policies to Emergency Response. 


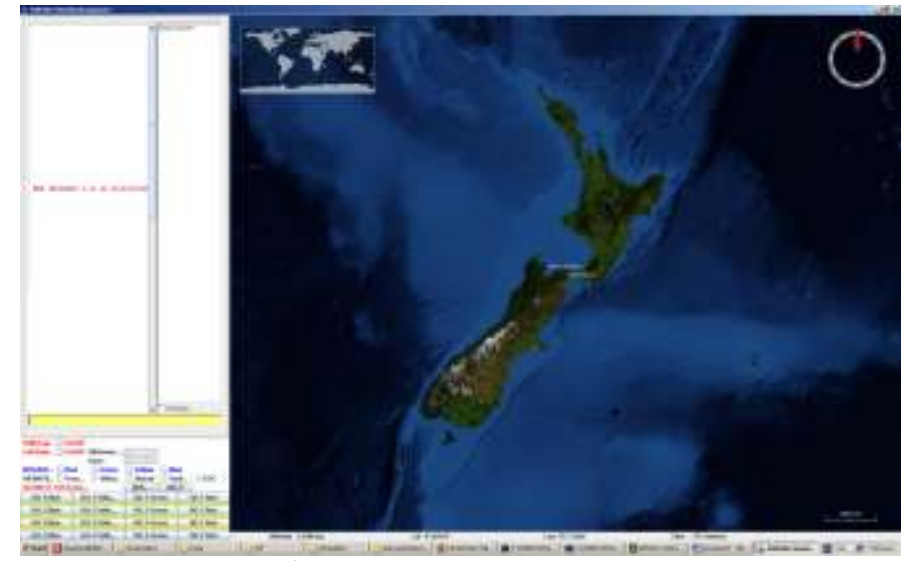

FIGURE 15. Layered Approach. This shows the first level view of our tool wherein the panel on the left-hand side allows the user to see the data and information that they need, selectively.

Fig. 15 shows the presentation of the top-level screen as the user enters the starting phase of the simulation. We take a layered approach by presenting the data and information, as the user sees fit, rather than all in one piece, as we did with CAVIAR. The user is notified with messages on the top-left panel and allowed to choose which of resources, incidents and cell phone data that they wish to see appearing on the screen. They may choose to see one or the other or some combination of this information, with the progress of time. We also add appropriate labels to indicate the actual location that the user is viewing in the OSM layer, as a reference point. This can be extended to mark administrative domains as well. A layered approach is a direct consequence of our modular framework.

The first level zoom in Reverse 111 shows a lot more information, including the scale $(20 \mathrm{~km})$ and the Latitude and Longitude where the zoom is occurring, as opposed to CAVIAR. This is important for user-orientation. Furthermore, this visualization environment takes into account the terrain, much better than using static maps, thereby making it easier for the user to identify valid paths and administrative domains. In our earlier examples, these were difficult to identify, specifically elevation, but that is shown to a greater detail in this environment. Fig. 16 shows the setting of administrative domains demarcated by the magenta lines. In this case, we have Christchurch, Rangiora, Oxford and Ashburton as the four different administrative domains that must interact and co-ordinate resources at the time of an emergency.

In this example we show the introduction of emergency events at an interval of 4 seconds each, indicated by the rhombus-shaped objects, with the four colors standing for one kind of static resource with numbers inside them indicating the resource-requirement. For example, if a rhombus appears with 3-yellow,0-red,1green and 1-blue, that is the resource requirement for that emergency, at the time it is occurring. This is shown in Fig. 16 upon zooming in on the resource. This requirement can dynamically change if a co-located cell- phone user, indicates as much back to the system. The resource requirements are important to assess the scheduling policy's action.

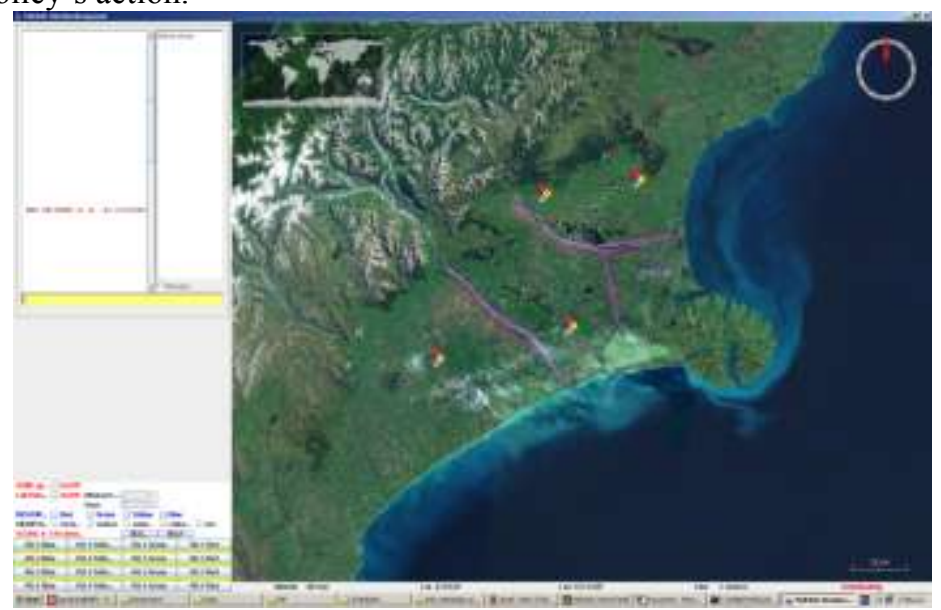

FIGURE 16. Administrative Domains. Four administrative domains, Christchurch, Oxford, Rangiora and Ashburton must act in tandem for this simulation situation.

Events occur at pre-determined locations or according to various pre-simulated scenarios.

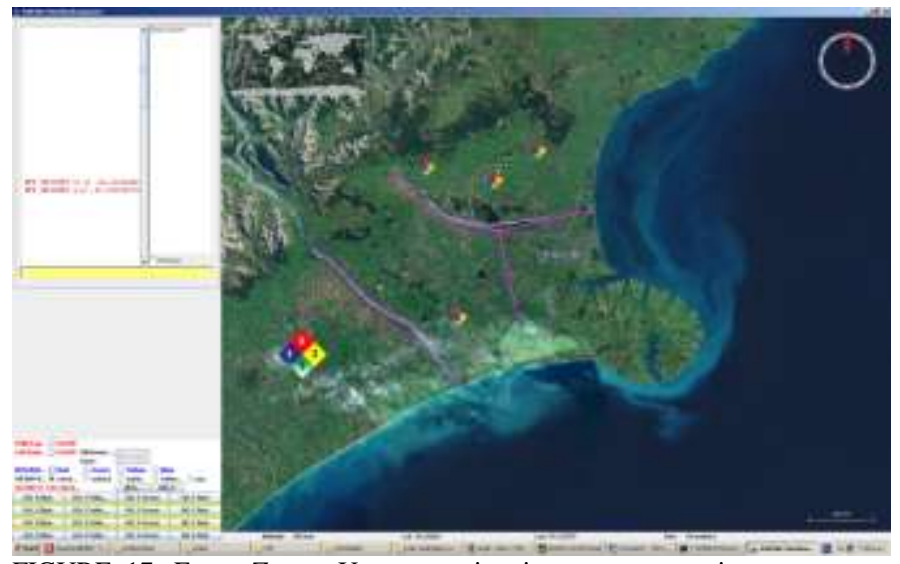

FIGURE 17. Event Zoom. Upon zooming in on an event, its resource requirements are shown to the user. In this case, the event required 4-blue resources, 3 yellow, 3 green and zero red resources.

While we take into account actual numbers of fire-trucks and other resources that have been reported on public websites accounting for the State Department's response to such activities, we combine this with an intuitive approach to where and how events within an emergency scenario might occur and our visual representation confirms that our placement of events is accurate. Fig. 18 shows the location of resources of a certain kind, when the user selects that resource should be shown on screen. The location of resources also gives the user a visual clue on the performance of our schedulers, when they try to pick the resource that is suited, according to their policy, to send to a particular event. Fig. 19 shows multiple resources 
(of various colours on the screen, as selected by the user. In this view, the user has also chosen to play as the administrative domain of Christchurch, therefore placing a greater emphasis on that region alone.
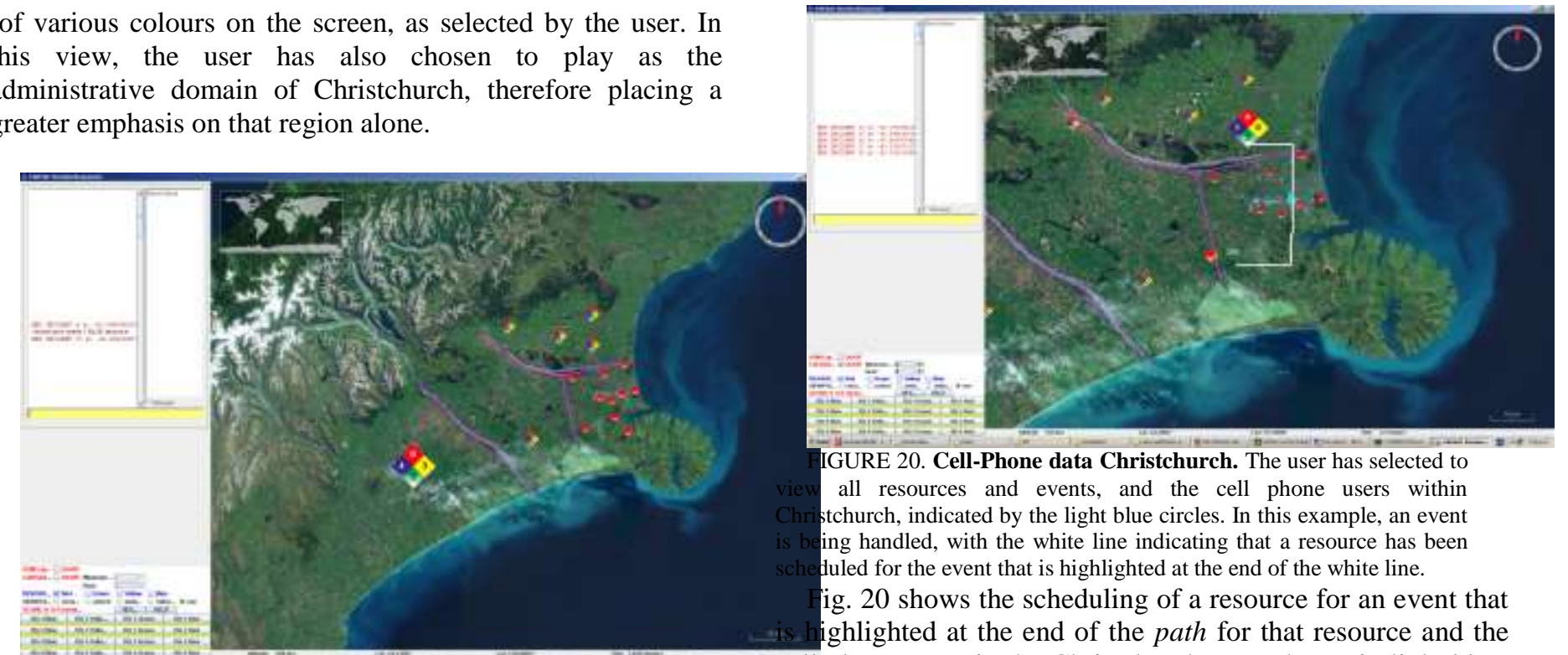

GURE 20. Cell-Phone data Christchurch. The user has selected to all resources and events, and the cell phone users within tchurch, indicated by the light blue circles. In this example, an event ng handled, with the white line indicating that a resource has been luled for the event that is highlighted at the end of the white line. ig. 20 shows the scheduling of a resource for an event that

FIGURE 18. Resource Layers. The user can select to see the actual physical location of resources, in this case they have chosen to see the location of all the red resources. These resources are applied to Emergency management in this example but can be easily extended to logistics and visualizing Supply chains.

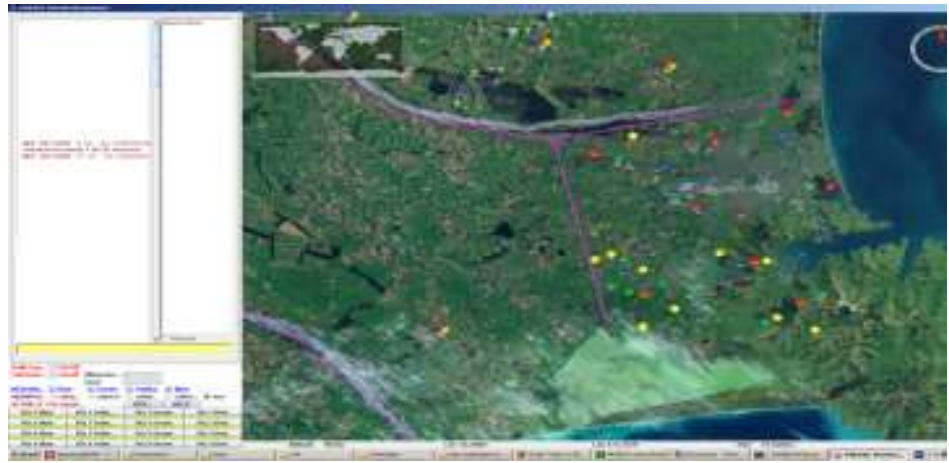

FIGURE 19. Resource Layers for Christchurch. The user has selected to view all resources and play as the administrative role of Christchurch, rather than all four domains indicated in earlier figures.

\section{B. Path Selection}

In this section, we discuss how paths are selected in order to get a resource to an event that requires it. n cell-phone users in the Christchurch area, shown in light blue.

Cell phone users are considered soft resources that provide information. In our prototype, the path selection process goes through the steps described in the flowchart shown in Fig. 21.

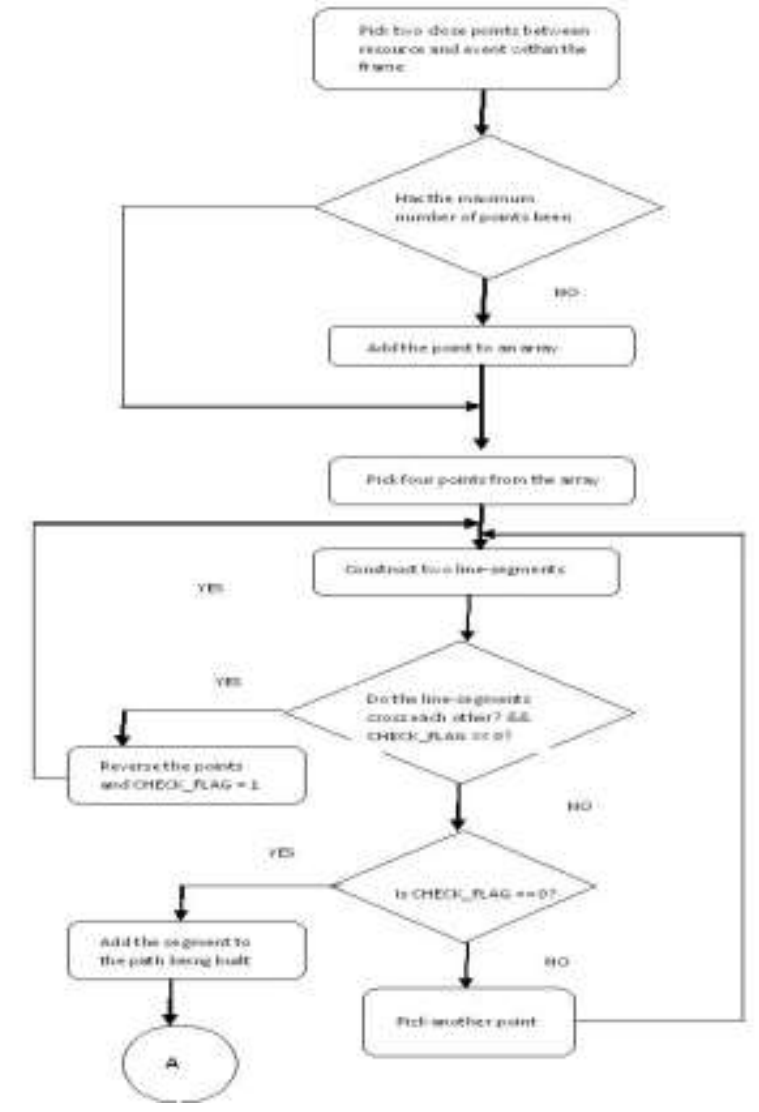

FIGURE 21. Building a Path. The system constructs a path in a piecewise fashion for each valid FRAME, which is only within the region that can be traversed without intervening geographic hindrances like water-bodies, etc. 
We draw the reader's attention to the fact that the user sets up the specific regions or frames that are valid for the resource to traverse. Our system constructs a piece-wise linear path, for each frame of valid points, located within a sub-region that can be navigated without any obstacles in the resource's way such as water-bodies or gorges (in the case of New Zealand's South Island). Fig. 21 shows the construction of the path, while ensuring that none of the segments cross each other, which would make for a circuitous route for the resource, as it reaches the event of interest.

Fig. 22 shows a co-located user, that is directly on the path being traversed, that can provide some insight into the situation towards which the resource is travelling. The user on this path has reputation information associated with them, besides actual physical location. For example, the user is associated with tower \#27, which has seen a total call volume of about 1000 calls, 276 from distinct callers. This tower is heavily loaded at the time of the emergency (we are tracking an hour of call-time in this example and filter only users that have not moved across cell-towers, indicating static users and not perpetrators that are fleeing) and it is likely that this user is reputable as the average call time of calls on this tower are about 22 seconds, indicating that most calls are being placed to check to see if the called party is safe.

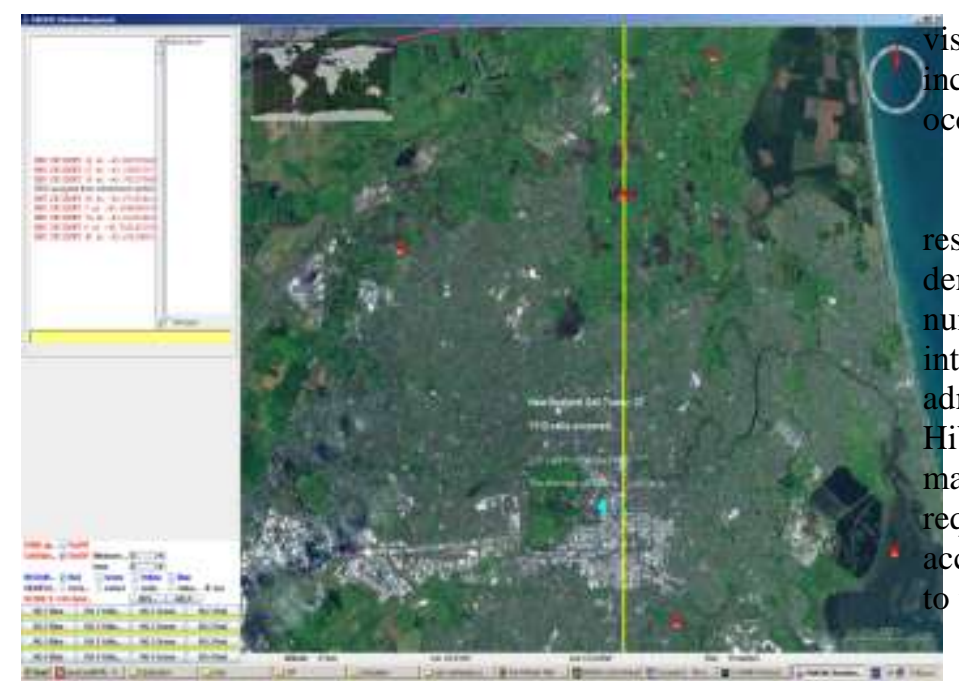

FIGURE 22 Location + Reputation = Information. This figure shows obtaining information from a reputed user, co-located with the emergency event and modifying the resource requirements to take that users-input into consideration.

\section{Scheduling Agents}

In this section, we perform a different evaluation of four scheduling agents, which was joint work with the University of Washington. We pick the three scenarios introduced in Fig. 17 , for this evaluation, in the greater Seattle region. The scale of emergencies in this region is different, as is our evaluation method, when compared to the result presented in Chapter 5.

In order to simulate multiple first responders within a response scenario, we added an agent class that simulates four different schedulers, greedy, lottery, round-robin and first-fit. In order to encode realistic scheduler behavior, we used a combination of resource-sharing policies, and resource and event selection strategies. These heuristics are easily extensible to encode newer policies and many more scheduler types, either distributed or otherwise.

The emergency response behavior of sub-classed agent types are varied by tweaking three parameters that affect agent behavior: (1) their policy on sharing resources with neighboring administrative domains (for example, does the mayor of Christchurch share with the mayor of Rangiora, etc.), (2) their strategy for determining which active unresolved incident should be attended to next (i.e does the scheduler choose the first event which appears or perform calculations to see which event can be responded to with the closest resources), and (3) their strategy for determining which available resource to assign the targeted incident (i.e, if an event requires 3 red resources, do we get all three to the event in one go or do we phase out the resource delivery).

Each agent takes a turn in effecting the dynamic sualization until the scheduled simulation time ends or all cidents have been handled and no more are scheduled to ccur. A turn is calculated as follows:

An agent figures out its resource surplus (how many sources it has free, minus the number of unmet resource mands from incidents in its jurisdiction). We call this umber $\mathbf{R}$ (note: $R$ is occasionally negative). We then terrogate the agent's R-level willingness to help other Iministrative domains. we call this number HiWat. If $\mathrm{R}>$ iWat, the agent looks at the requests for help that have been ade by other players (human or agent). If there is an open quest and the $\mathrm{R}>\mathrm{HiWat}$, the agent chooses a resource cording to its Resource Selection Strategy (RSS) and send it the incident specified in the request. This ends a turn.

If instead R <= HiWat, we interrogate the agent's R-level willingness to ask for help from other administrative domains. we call this number LoWat. If $\mathrm{R}<$ LoWat, the agent chooses the last incident on our priority list (if there is one; priority as determined by our ISS) and broadcasts a request for help on that incident. This ends a turn.

If instead $\mathrm{R}>=$ LoWat, the agent chooses an incident according to its Incident Selection Strategy (ISS) subject to its Resource Sharing Policy (RSP). This RSP provides a primary ordering of the incidents according to jurisdiction, the ISS provides a secondary ordering in case of ties (and usually we 
find there are many ties). If there are no incidents to choose, this ends a turn.

The agent chooses a resource to send to the incident it has chosen by following its embedded heuristics, which in this case are according to an RSS. The agent sends the resource to the incident it chose in step 4. This ends a turn.

For the study we document in this thesis, the agent behaviordefining parameters we chose to test include:

- $\quad$ HiWat - an integer representing willingness to give help when asked.

- $\quad$ LoWat - an integer representing willingness to ask for help

- $\quad$ Resource Sharing Policy (RSP) - one of 5 alternatives representing willingness to volunteer help.

- Incident Selection Strategy (ISS) - one of 4 alternatives representing heuristic for choosing the next incident to apply resources.

- $\quad$ Resource Selection Strategy (RSS) - one of 2 alternatives representing the heuristic for choosing the next resource to assign.

\section{Resource Sharing Policy}

An agent's RSS policy describes under what conditions it will voluntarily respond to incidents outside its given geographic region. Five policies are defined and implemented in agent sub-classes. we refer to the policies by name in order to assist reading comprehension when describing our experiments in a later section:

- Sociopath - Never volunteer aid to another
region.
$-\quad$ Selfish - Prefers incidents in its own region, but will volunteer aid to another region if there are no active incidents in its own region.

- Equalitarian - Does not take geographic region into account when determining which incident to handle next.

- $\quad$ Selfless - Prefers to volunteer for incidents in another region, but will handle incidents in its own region if there are no outside incidents to handle.

- $\quad$ Altruist - Never handles its own incidents, but will always volunteer for incidents outside its region.

\section{E. Incident Selection Strategy}

Within the broader resource-sharing policy, there is still the question of which incident to handle first, since there might be many active incidents within a single geographic region at any given time. Which incident is selected in the end depends first on policy and then on incident selection strategy. we implemented four representative incident selection strategies for our experiments:

- First Fit - Chooses the incident with the lowest incident number regardless of other considerations. Computationally, this is far the simplest of the strategies.

- $\quad$ Round Robin - Chooses the incident that has been active the longest.

- $\quad$ Lottery - Gives each incident a number of tickets equal to the total number of resources it requires, and chooses a ticket at random. The incident holding the winning ticket is selected.

- Greedy - Considers the resources that would have to be applied to each incident, and chooses the incident that could be handled most quickly (that is, on the basis of the furthest required resource).

\section{F. Resource Selection Strategy}

Once an incident has been identified, an agent must choose resources to assign to that incident in order to assist in incident resolution. There are likewise many possible strategies for choosing between resources to assign. For the demonstration purposes of this thesis, we encoded two resource selection strategies:

First Fit - Chooses the free resource with the lowest resource number.

Closest - Chooses the free resources closest to the incident.

$$
\text { G.Agent Types }
$$

The agent types that we use to demonstrate our software use are a combination of the elements above, particularly the two selection strategies, since the policy can be supplied as a parameter for every agent type:

- LocalAgent: First Fit incident selection, First Fit resource selection.

- RoundRobinAgent: Round Robin incident, Closest resource.

- LotteryAgent: Lottery incident, Closest resource.

- GreedyAgent: Greedy incident, Closest resource.

\section{Simulation RunS}

Each of the scenarios is divided into the same four geographic areas to represent administrative domains for the emergency response agents. we varied all possible 160 agent characteristic mixes across all four geographic regions to create 640 runs for each scenario. we watched each of the 640 
simulations take place for each scenario and noticed many interesting patterns of resource allocations including obvious inefficient motions for inferior characteristic sets. The response activity across the two major bridges was especially interesting to watch as were the clustering of movements at times between administrative domains. Figure 23looks at the time to completion for each simulation across all three scenarios. Scenario completion times include the area from the top of each scenario area down to 0 on the $\mathrm{x}$-axis. In all but twelve of the 640 runs, the tsunami scenario took less time to mitigate than the others (in twelve runs the completion time was identical to the earthquake scenario). The earthquake scenario took less time than the bioweapon scenario in all cases (but was much closer in the most efficient cases). The distribution of response times gives a sense of how significant agent behavior mix was to the completion time of the scenario. The more isolated the community event to one jurisdiction, the more impact agent behavior makes on completion time.

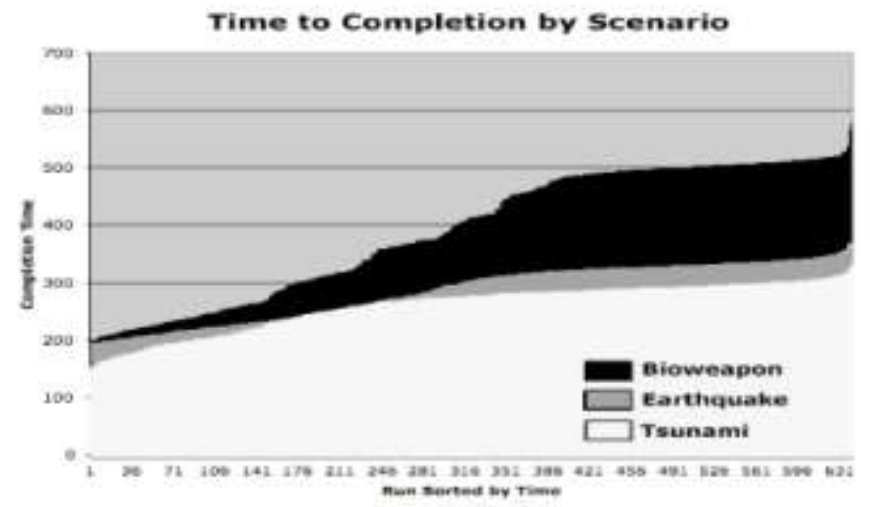

FIGURE 23 Seattle results. This figure shows our running various agents within the greater Seattle region. we do not take into account co-located callers in this example.

\section{CONCLUSIONS}

CAVIAR was our initial prototype, which was very static in its definition of events, resources and crowds. We mapped caller traces obtained from Telecom New Zealand's network wherein the user's movement was tracked across cell-towers at which the calls originated and terminated. Events were selected across special event days such as Rugby Game days where the call-traffic patterns and user-movement patterns changed (and demonstrated crowding at certain locations downtown or close to the stadium). We evolved this prototype to accommodate four guiding design principles for efficient design of a test-bed for Emergency Management Simulation including the incorporation of an end-to-end design, incorporation of a number of governmental and nongovernmental entities and policies, efficient, inter-operable communication between first responders and the ability to change response parameters with live feedback. We evaluated the performance of our prototype using 640 simulation runs and presented these results to evaluate various approaches to live emergency response.

\section{ACKNOWLEDGMENT}

We acknowledge the work of Konrad Schroder on help with building agents and enabling us to test these effectively.

\section{REFERENCES}

1. Ito, A., Murakami, H., Watanabe, Y., Fujii, M., Yabe, T., Haraguchi, Y., Tomoy-asu, Y., Kakuda, Y., Ohta, T., Hiramatsu, Y.: Universal use of information delivery and display system using ad hoc network for deaf people in times of disaster. (2008) 486-491

2. Wu, W., Cao, J., Zheng, Y., Zheng, Y.P.: Waiter: A wearable personal healthcare and emergency aid system. (2008) 680-685

3. Lee, R.G., Chen, K.C., Hsiao, C.C., Tseng, C.L.: A mobile care system with alert mechanism. Information Technology in Biomedicine, IEEE Transactions on 11(5) (2007) 507-517

4. Inoue, Y., Sashima, A., Ikeda, T., Kurumatani, K.: Indoor emergency evacuation service on autonomous navigation system using mobile phone. (2008) 79-85

5. Filippoupolitis, A., Hey, L., Loukas, G., Gelenbe, E., Timotheou, S.: Emergency response simulation using wireless sensor networks. In: Ambi-Sys '08: Proceedings of the 1st international conference on Ambient media and systems, ICST, Brussels, Belgium, Belgium, ICST (Institute for Computer Sciences, SocialInformatics and Telecommunications Engineering) (2008)

6. Chitumalla, P., Harris, D., Thuraisingham, B., Khan, L.: Emergency response applications: Dynamic plume modeling and real-time routing. Internet Computing, IEEE 12(1) (2008) 38-44

7. Basu, S.N.: Fermi 2 emergency response information system. Energy conversion, ieee transactions on EC-1(2) (1986) 16-22

8. Serpa, D.P., Walker, D.M., Jenckes, T.A.: Emergency monitoring, assessment and response system for diablo canyon nuclear power plant. Nuclear Science, IEEE Transactions on 28(1) (1981) 236241

9. Burnett, B., Bowering, G., Hagelstrom, R., Tate, D., Ramanujan, R.: Multi-radio nodes for mobile mesh networks for emergency response and battle command systems. (2007) 1-7

10. Symons, L., Pavia, R., Hodges, M.: Emergency response in national marine sanctuaries. (2005) 345-351 Vol. 1

11. Gertman, D.I., Bruemmer, D.J., Scott Hartley, R.: Improving emergency response and human- robotic performance. (2007) 334-340

12. Bansal, A., Patel, K., Gupta, G., Raghavachari, B., Harris, E., Staves, J.: Towards intelligent services: a case study in chemical emergency response. (2005) -758

13. Kung, H.Y., Ku, H.H., Wu, C.I., Lin, C.Y.: Intelligent and situation-aware pervasive system to support debris-flow disaster prediction and alerting in Taiwan. J. Netw. Comput. Appl. 31(1) (2008) $1-18$

14. Miyauchi, K., Yonezawa, Y., Ogawa, H., Maki, H., Caldwell, W.: A mobile phone-based safety and life support system for elderly people. (2005) 81-84

15. Pries, R., Hobfeld, T., Tran-Gia, P.: On the suitability of the short message service for emergency warning systems. Volume 2. (2006) 991-995

16. Drane, C., Macnaughtan, M., Scott, C.: Positioning gsm telephones. Communications Magazine, IEEE 36(4) (1998) 46-54, 59

17. Scott Shane, E.L.: Stumbling storm-aid effort put tons of ice on trips to nowhere. The New York Times (2005)

18. Campbell, B., Schroder, K. (2009) Training for emergency response with RimSim:Response!, In Proceedings for the Defense, Security, and Sensing 2009 Conference (Orlando, FL). 
19. Filippoupolitis, A., Hey, L., Loukas, G., Gelenbe, E., Timotheou, S.: Emergency response simulation using wireless sensor networks. In: Ambi-Sys '08: Proceedings of the 1st international conference on Ambient media and systems, ICST, Brussels, Belgium, Belgium, ICST (2008)

20. Chitumalla, P., Harris, D., Thuraisingham, B., Khan, L.: Emergency response applications: Dynamic plume modeling and real-time routing. Internet Computing, IEEE 12(1) (2008) 38-44

21. Carl A. Waldspurger. Lottery and Stride Scheduling: Flexible Proportional-Share Resource Management, Ph.D. dissertation, Massachusetts Institute of Technology, September 1995.

22. Yishay Mansour, Boaz Patt-Shamir, Greedy Packet Scheduling on Shortest Paths (1991), Journal of Algorithms

23. Rubén Ruiz, Thomas Stützle, A simple and effective iterated greedy algorithm for the permutation flowshop scheduling problem (2006), European Journal of Operational Research

24. Linehan et. Al., Developing a serious game to evaluate and train group decision making skills, Proceedings of the 13th International MindTrek Conference: Everyday Life in the Ubiquitous Era, P106-113, 2009.

25. Fortes, Macintosh, Effective citizen relationship management: Hurricane Wilma and Miami-Dade County 311, Proceedings of the 2006 international conference on Digital government research , Pages: 452 - 453 , 2006 , San Diego, California

26. Artigas et. $\mathrm{Al}, \boldsymbol{A}$ regional approach to geographic information sharing in northern New Jersey, USA, Proceedings of the 2008 international conference on Digital government research, Montreal, Canada, P: 132-136, 2008.

27. L. Frishberg, Looking back at plan AHEAD ${ }^{\mathrm{TM}}$ : exercising usercentered design in emergency management, Conference on Human Factors in Computing Systems, Portland, OR, 2005

28. Boukerche, et. Al., Web-Based Distributed Simulations Visualization and Control with HLA and Web Services, Proceedings of the 2008 12th IEEE/ACM International Symposium on Distributed Simulation and Real-Time Applications, P 17-23, 2008

29. $\mathrm{R}$. Woltjer et. $\mathrm{Al}$, Role-playing exercises to strengthen the resilience of command and control systems, Proceedings of the 13th Eurpoean conference on Cognitive ergonomics: trust and control in complex socio-technical systems, ACM International Conference Proceeding Series; Vol. 250 P71-78, 2006.

30. Artigas et. Al., Real-time ocean surge warning system, meadowlands district of New Jersey, Proceedings of the 10th Annual International Conference on Digital Government Research: Social Networks: Making Connections between Citizens, Data and Government, P216-222, 2009.

31. D. Tarchi, et. Al., The communication infrastructure for emergency management: the In.Sy.Eme. vision, Proceedings of the 2009 International Conference on Wireless Communications and Mobile Computing: Connecting the World Wirelessly, Leipzig, Germany 2009.
32. T. Clark, C. Waring, A simulation approach to analysis of emergency services and trauma center management, Proceedings of the 19th conference on Winter simulation, Atlanta, Georgia, United States, 1987.

33. W. Bostian et. Al., Testbed for High-Speed 'End-to-End' Communications in Support of Comprehensive Emergency Management, Proceedings of the 2004 annual national conference on Digital government research, Seattle, WA.

34. L. Paylen et. Al., A vision for technology-mediated support for public participation \& assistance in mass emergencies \& disasters, Proceedings of the 2010 ACM-BCS Visions of Computer Science Conference, Edinburgh, United Kingdom.

35. Mendonca et. Al., Collaborative adhocracies and mix-and-match technologies in emergency management, Communications of the ACM, SPECIAL SECTION: Emergency response information systems: emerging trends and technologies,P44-47, 2007.

36. $\mathrm{N}$ Adam et. Al, Secure information sharing and analysis for effective emergency management, Proceedings of the 2008 international conference on Digital government research, Montreal, Canada.

37. $\mathrm{Wu}, \mathrm{X}$. Zhang, Supporting collaborative sensemaking in mapbased emergency management and planning, Proceedings of the ACM 2009 international conference on Supporting group work, Sanibel Island, Florida, USA.

38. Riberio, A. Ferworn, Computational public safety in emergency management communications, Proceedings of the 6th International Wireless Communications and Mobile Computing Conference, Caen, France, 2010.

39. N. M. Patrikalakis, J. J. McCarthy, et al., “Towards a Dynamic Data Driven System for Rapid Adaptive Interdisciplinary Ocean Forecasting,” In Dynamic Data Driven Application Systems, F. Darema, ed., Kluwer Academic Publishers, Netherlands, 2004.

40. Vaidyanathan, A., Johnston, D. (in press). The use of cell-phone technology for improved disaster response: A case-study from New Zealand. The Australasian Journal of Disaster and Trauma Studies.

41. Vaidyanathan, A. "Emergency Response with Proactive CrowdSourcing-Reverse-111", 5th International Conference on Critical Response Information Infrastructure Security (CRITIS), Athens, Greece, September 2010

\section{AUTHORS PROFILE}

Dr. Anu Vaidyanathan is Visiting Faculty at the Department of Computer Science and Engineering at IIT Ropar. Prior to joining IIT Ropar, Anu was the CEO of PatNMarks, the first Indian company to offer Value Added Services in the area of Intellectual Property. Anu graduated with a B.S in Computer Engineering from Purdue University in 2001. After getting a Masters in Computer Engineering from North Carolina in 2002 and a brief stint at IBM Austin, Anu finished her $\mathrm{PhD}$ in Electrical and Computer Engineering at the University of Canterbury at Christchurch, graduating in a record time of 26 months 